\title{
THE ABUNDANCES OF ISOTOPES IN THE COSMIC RADIATION
}

\author{
R. A. Mewaidt \\ California Institute of Technology Pasadena, CA 91125
}

\begin{abstract}
Studies of the isotopic composition of nuclei in the cosmic radiation are reviewed, including abundances of the isotopes of elements from $\mathrm{H}$ to $\mathrm{Ni}$ (nuclear charge $1 \leq \mathrm{Z} \leq 28$ ), and their implications for cosmic ray origin, acceleration, and transport in the Galaxy. The review focuses on determinations of the composition of cosmic ray source material, and the extent to which the isotopic composition of this material is different from, or similar to, typical solar system material and other samples of Galactic matter. Theoretical models that have been advanced to explain the observed overabundance of neutron-rich isotopes in cosmic rays are described. Also discussed are studies of various radioactive "clocks" that record the time-scales associated with the nucleosynthesis, acceleration, and transport of cosmic ray nuclei, and studies of the so-called "anomalous" cosmic ray component, thought to represent a sample of the neutral interstellar medium. Finally, the goals and prospects for future cosmic ray isotope spectrometers are described.
\end{abstract}

\section{INTRODUCTION}

In their papers ${ }^{1,2,3}$ announcing the discovery of heavy cosmic ray nuclei Freier and co-workers commented that the relative abundances of $\mathrm{H}, \mathrm{He}$, and heavier nuclei that they observed in the cosmic radiation were generally consistent with what was then known about the abundances of elements in the Galaxy as a whole. They therefore anticipated that cosmic ray nuclei could open up a new window onto the study of the composition of matter in the galaxy. We now have quite accurate measurements of the composition of a wide range of elements in cosmic rays $^{4,5}$, and it has been shown that the atomic properties of the elements play an important role in shaping this composition, possibly through their first ionization potential $^{6,7}$.

As the techniques of cosmic ray spectroscopy have improved, especially over the past decade or so, it has become possible to study the isotopic composition of heavy cosmic ray nuclei, thereby shedding light on their nuclear history, including their synthesis in stars, and their subsequent interactions with the interstellar medium. This paper reviews what has been learned about the isotopic composition of cosmic rays to date, summarizes models for its interpretation, and discusses some of the prospects for extending these studies in the future.

There are a wide range of scientific objectives that cosmic ray isotope measurements can address, including studies of: nucleosynthesis in nearby regions of the Galaxy; the chemical evolution of the Galaxy; the time delay between nucleosynthesis and cosmic ray acceleration; cosmic ray reacceleration by supernova shocks; and the storage-time and pathlength distribution of cosmic rays in the Galaxy. The primary objective of this conference is to characterize and intercompare the 
element and isotope abundance patterns in various samples of matter in the universe. This paper will therefore focus on the isotopic composition of cosmic ray sources, which provide us with a sample of matter from outside the solar system in the form of "primary" cosmic rays. However, in order to interpret the abundances of this sample of extra-solar matter it is also necessary to understand the history of cosmic rays since the time of their acceleration, as revealed by studies of so-called "secondary" cosmic rays produced by the breakup of heavier nuclei in collisions with interstellar or other material.

\section{COSMIC RAY CLOCKS AND SECONDARY NUCLEI}

It is generally accepted that typical cosmic ray nuclei with energies below a few $\mathrm{GeV} / \mathrm{nucleon}$ have traversed an average of 6 to $9 \mathrm{~g} / \mathrm{cm}^{2}$ of material during their lifetime, as evidenced by the abundances of secondary nuclei such as $\mathrm{Li}, \mathrm{Be}$, and $B$ in cosmic rays. This pathlength (which is actually the mean of a pathlength distribution, and is known to be energy dependent), is usually interpreted as the mean free path for the escape (or "leakage") of cosmic rays from the galaxy ${ }^{8,9}$. Studies of secondary nuclei in different parts of the periodic table can reveal whether their parents have shared a common history.

Cosmic Ray ${ }^{2} \mathrm{H}$ and ${ }^{3} \mathrm{He}$ : The rare isotopes ${ }^{2} \mathrm{H}$ and ${ }^{3} \mathrm{He}$ in cosmic rays are believed to be of secondary origin, produced mainly by the breakup of primary cosmic ray ${ }^{4} \mathrm{He}$. Although the four stable $\mathrm{H}$ and $\mathrm{He}$ isotopes $\left({ }^{1} \mathrm{H},{ }^{2} \mathrm{H},{ }^{3} \mathrm{He}\right.$ and $\left.{ }^{4} \mathrm{He}\right)$ were historically the first to be resolved in the cosmic radiation, their relative abundances have to date been measured over only a limited energy interval. There has recently been renewed interest in the abundance of ${ }^{2} \mathrm{H}$ and ${ }^{3} \mathrm{He}$ in cosmic rays because of indications that primary $\mathrm{H}$ and $\mathrm{He}$ may have had a different history from that of heavier cosmic rays. For example, there is evidence for an overabundance of antiprotons ${ }^{10,11}$ and positrons ${ }^{12-14}$ in cosmic rays (two secondary species produced by nuclear collisions of primary protons and alpha particles), and it has also been suggested that the energy spectra of $\mathrm{H}$ and possibly He differs from that of heavier cosmic ray nuclei ${ }^{15}$.

Figure 1. shows selected solar minimum measurements of the ${ }^{2} \mathrm{H} /{ }^{4} \mathrm{He}$ and ${ }^{3} \mathrm{He} /{ }^{4} \mathrm{He}$ ratios along with calculations ${ }^{16}$ of these ratios. The solid curves are for a pathlength distribution ${ }^{17}$ that fits measurements of heavier cosmic ray secondary/primary ratios such as $\mathrm{B} / \mathrm{C}$. Note that below $\sim 80 \mathrm{MeV} /$ nucleon for ${ }^{2} \mathrm{H}$ and $\sim 100 \mathrm{MeV} /$ nucleon for ${ }^{3} \mathrm{He}$, where the measurements are made by satellite instruments, the measurements of both ratios are generally consistent with the standard rigidity-dependent "Leaky Box" model. The only clear exception is the balloon measurement of ${ }^{2} \mathrm{H}$ at $\sim 80$ to $150 \mathrm{MeV} /$ nucleon $^{18}$, which is difficult to reconcile with conventional cosmic ray propagation models, and with lower energy satellite data.

The recent antiproton observations have led to several new cosmic ray origin and/or propagation models in which cosmic rays from some sources have traversed a great deal of matter, possibly material surrounding cosmic ray acceleration 
Figure 1: Measured and calculated ${ }^{2} \mathrm{H} /{ }^{4} \mathrm{He}$ and ${ }^{3} \mathrm{He} /{ }^{4} \mathrm{He}$ ratios. The solid curves ${ }^{16}$ are for a standard "leaky-box" model including solar modulation effects. The dashed curve (Cowsik and Gaisser model ${ }^{21}$ ), assumes $30 \%$ of cosmic rays originate in sources surrounded by $\mathbf{5 0}$ $\mathrm{g} / \mathrm{cm}^{2}$. Only selected solar minimum satellite ${ }^{16,23-26,114}$ and balloon ${ }^{18,27-30}$ data are shown below 500 $\mathrm{MeV} /$ nucleon. The ${ }^{3} \mathrm{He} /{ }^{4} \mathrm{He}$ upper limit at $6 \mathrm{GeV} / \mathrm{nucleon}$ is a reinterpretation $^{19}$ of a recent balloon measurement ${ }^{22}$, while the ${ }^{2} \mathrm{H} /{ }^{4} \mathrm{He}$ point at $1 \mathrm{GeV} /$ nucleon $^{29}$ is from Webber $^{30}$. The low energy observations have been corrected for anomalous ${ }^{4} \mathrm{He}$. The balloon observations of ${ }^{3} \mathrm{He}$ have been corrected for ${ }^{4} \mathrm{He}$ fragmentation in the atmo sphere ${ }^{16}$, but the ${ }^{2} \mathrm{H}$ observations have not ${ }^{118}$. For earlier ${ }^{2} \mathrm{H}$ and ${ }^{3} \mathrm{He}$ data see Meyer ${ }^{20}$ and Beatty ${ }^{26}$.

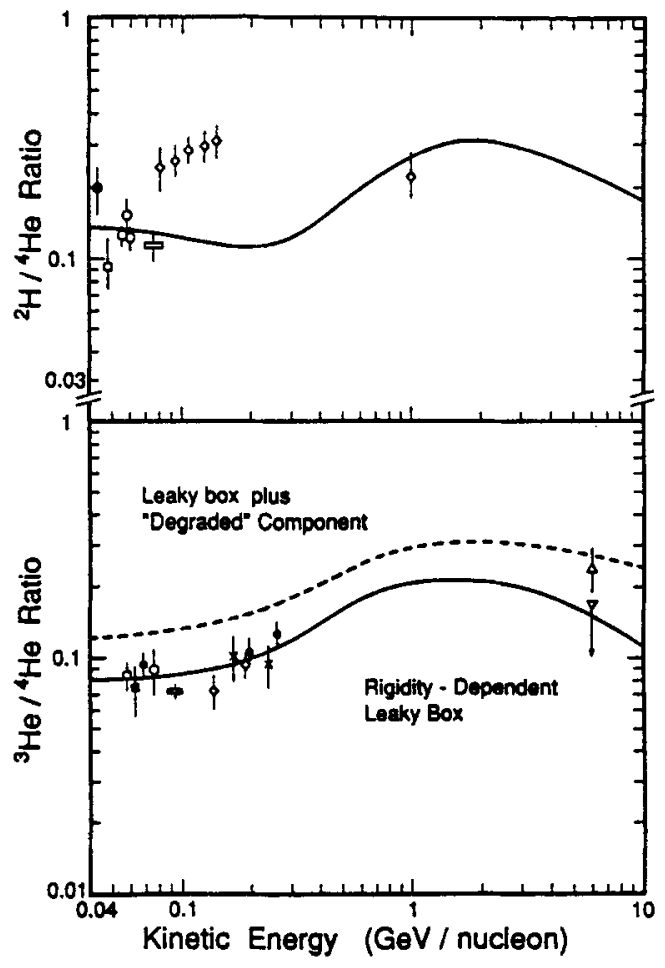

sites ${ }^{21,31-35}$. Most such models also lead to an excess of ${ }^{2} \mathrm{H}$ and ${ }^{3} \mathrm{He}$ in addition to antiprotons and positrons. As an example, Figure 1 includes the ${ }^{3} \mathrm{He} /{ }^{4} \mathrm{He}$ ratio expected from the model of Cowsik and Gaisser ${ }^{21}$ in which a "degraded" component of cosmic rays originates in "thick" sources surrounded by $\sim 50 \mathrm{~g} / \mathrm{cm}^{2}$ of material. A similar excess is expected for ${ }^{2} \mathrm{H}$. Note that with the exception of the ${ }^{2} \mathrm{H}$ measurements at $\sim 100 \mathrm{MeV} /$ nucleon ${ }^{18}$, there is no evidence from ${ }^{2} \mathrm{H}$ and ${ }^{3} \mathrm{He}$ to support these models, and the majority of the ${ }^{2} \mathrm{H}$ and ${ }^{3} \mathrm{He}$ observations therefore significantly restrict possible interpretations of the observed overabundance of antiprotons. It should be remembered, however, that there are almost no ${ }^{2} \mathrm{H}$ and ${ }^{3} \mathrm{He}$ observations at energies of several $\mathrm{GeV} / \mathrm{nucleon}$ and above, where secondary antiprotons are produced. This will be an area of increased activity during the next few years as new balloon-borne magnet instruments by a number of groups extend these observations to higher energies.

Cosmic Ray Clocka: Among the isotopes that are produced as "secondaries" by the fragmentation of heavier cosmic rays are a number which are radioactive, with half-lives suitable for measuring the average lifetime of cosmic ray nuclei in the galaxy. Examples are: ${ }^{10} \mathrm{Be}\left(\mathrm{t}_{1}=1.6 \times 10^{6} \mathrm{yr}\right),{ }^{14} \mathrm{C}\left(\mathrm{t}_{\mathrm{t}_{3}}=5730 \mathrm{yr}\right),{ }^{26} \mathrm{Al}\left(\mathrm{t}_{\mathrm{H}_{\mathrm{H}}}\right.$ $\left.=9 \times 10^{5} \mathrm{yr}\right),{ }^{36} \mathrm{Cl}\left(\mathrm{t}_{\mathrm{k}}=3 \times 10^{5} \mathrm{yr}\right)$ and ${ }^{54} \mathrm{Mn}\left(\mathrm{t}_{\mathrm{k} / \mathrm{e}}\right.$ estimated ${ }^{36}$ to be $\left.\sim 2 \times 10^{6} \mathrm{yr}\right)$. Since these nuclei would not be expected to be present in any significant amount in cosmic ray source material, their relative abundance in cosmic rays is a calculable 
function of the amount of material traversed by heavier nuclei and the mean lifetime since acceleration. Figure 2 compares measurements of two of these clocks, ${ }^{10} \mathrm{Be}$ and ${ }^{26} \mathrm{Al}$, with calculations parameterized by the average density of material in the propagation region. Note that both the ${ }^{10} \mathrm{Be}$ and ${ }^{26} \mathrm{Al}$ abundances are consistent with a mean density of $\sim 0.2$ atoms $/ \mathrm{cm}^{3}$, considerably less than the average density of matter in the galactic plane $\left(\sim 1\right.$ atoms $\left./ \mathrm{cm}^{3}\right)$. For a mean pathlength for escape from the galaxy of $\sim 6 \mathrm{~g} / \mathrm{cm}^{2}$ (appropriate to $\sim 100-300$ $\mathrm{MeV} / \mathrm{nucleon}$, where the best present measurements are), this corresponds to a lifetime of $\sim 10-15$ million years ${ }^{38,40,41}$. This age estimate implies that cosmic rays most likely represent a much younger sample of material than the solar system, which formed $\sim 4.6 \times 10^{9}$ years ago. As Figure 2 indicates, there is considerable energy dependence expected for both the ${ }^{26} \mathrm{Al} /{ }^{27} \mathrm{Al}$ and ${ }^{10} \mathrm{Be} /{ }^{9} \mathrm{Be}$ ratios as a result of time-dilation effects and the energy dependence of the production cross-sections.

Figure 2: Calculations of the ${ }^{10} \mathrm{Be} /{ }^{9} \mathrm{Be}$ and ${ }^{26} \mathrm{Al} /{ }^{27} \mathrm{Al}$ ratios vs. energy per nucleon (by Guzik and Wefel ${ }^{37}$ ), parameterized by the density of the propagation region in atoms per $\mathrm{cm}^{3}$, are compared with measurements by the Berkeley ${ }^{38,39}$ (open square), Chicago ${ }^{40,41}$ (closed circle), Goddard $^{42}$ (upward triangle), Minnesota ${ }^{43}$ (diamond), and New Hampshire ${ }^{44-47}$ (downward triangles) groups. The curves labeled $10^{9}$ show the expected result if there were no radioactive decay.

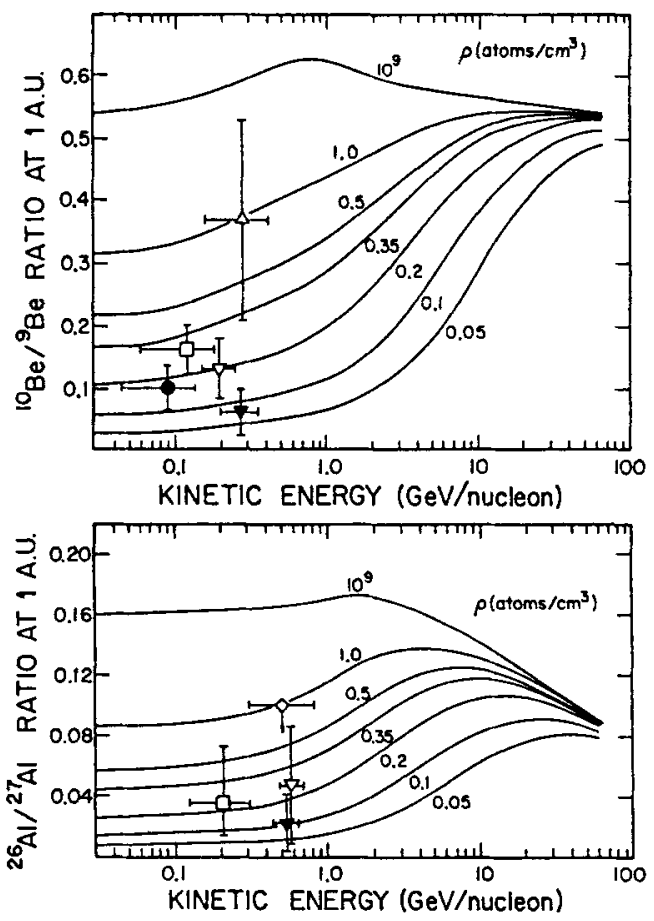

In addition, measurements of cosmic ray clocks with different half-lives can in principle measure the distribution of the material traversed by cosmic rays $39,48,49$. For example, if much of this material immediately surrounds cosmic ray sources we would expect a relatively greater production of radioactive secondaries in the distant past, so that the ratio of long-lived species to short-lived species should be enhanced. At the other extreme, the well-known radioactive isotope ${ }^{14} \mathrm{C}$ (half-life $=5730$ years) would be expected to have a measureable abundance in cosmic rays that would allow a comparison of the density of material traversed over the past $\sim 10^{4}$ years with the average density. 


\section{ISOTOPIC COMPOSITION OF COSMIC RAY SOURCE MATERIAL}

Figures 3 through 10 summarize selected measurements of the galactic cosmic ray source (GCRS) composition for 8 different elements. In each case the reported determinations are compared with the solar system (SS) composition compiled by Anders and Ebihara ${ }^{50}$, as well as other relevant measurements.

Neon (Figure 5) was the first element for which an anomalous isotopic composition was discovered ${ }^{70,64,61,57,73}$ and almost all recent measurements now agree that there is a large excess of ${ }^{22} \mathrm{Ne}$ in cosmic rays. However, the magnitude of that excess relative to the solar system composition is uncertain because the ${ }^{22} \mathrm{Ne} /{ }^{20} \mathrm{Ne}$ ratio in the solar system is a subject of controversy. Thus, for example, Cameron ${ }^{82}$ adopts the meteoritic component neon- $\mathrm{A}\left({ }^{22} \mathrm{Ne} /{ }^{20} \mathrm{Ne}=0.122\right)$ for his solar system standard, while Anders and Ebihara adopt the solar wind value of ${ }^{22} \mathrm{Ne} /{ }^{20} \mathrm{Ne}=0.073$. Thus GCRS ${ }^{22} \mathrm{Ne}$ is enhanced by anywhere from a factor of $\sim 3.5$ to 5.5 .

The various reported studies also generally agree that both ${ }^{25} \mathrm{Mg}$ and ${ }^{26} \mathrm{Mg}$ are overabundant by a factor of $\sim 1.5^{61,57,73,47,71}$ (see Figure 6). For Si (Figure 7) there is also evidence ${ }^{56}$ for a $\sim 50 \%$ excess of both neutron-rich isotopes from the Berkeley ISEE-3 instrument (which has the smallest uncertainties). The Caltech ${ }^{61}$ and HEAO-3 ${ }^{71,72}$ results also favor an excess, but the large uncertainties on these measurements include the solar system value. The recent balloon measurements reported by the UNH group ${ }^{46}$ have considerably smaller ${ }^{29} \mathrm{Si}$ and ${ }^{30} \mathrm{Si}$ abundances, also with sizeable uncertainties. Thus the Berkeley silicon results are still in need of confirmation.

Carbon and Oxygen are especially interesting because there are other measurements of their isotope abundances in other parts of the galaxy. Thus Hawkins and Jura ${ }^{51}$ have recently reported optical measurements towards five stars in four different directions that give a ${ }^{13} \mathrm{C} /{ }^{12} \mathrm{C}$ ratio in the local interstellar medium that is a factor of $2.1 \pm 0.2$ greater than the solar system value. There is also evidence for such differences in both the $C$ and $O$ isotope abundances measured by millimeter-wave techniques for both the galactic plane and the galactic center ${ }^{52,53}$. Unfortunately, both the ${ }^{13} \mathrm{C}$ and ${ }^{18} \mathrm{O}$ abundances in cosmic rays have large secondary contributions. As a result, when the uncertainties in presently available cross sections are taken in to account ${ }^{83}$ the measurements are consistent with the solar system ratio of ${ }^{13} \mathrm{C} /{ }^{12} \mathrm{C}=0.011$.

$\mathrm{Fe}$ has long been regarded as a key element for understanding the nucleosynthesis of cosmic ray material ${ }^{84,85}$. Although ${ }^{54} \mathrm{Fe}$ has been identified in cosmic rays ${ }^{62,75}$ and its source abundance is apparently within a factor of two of the solar system value, there are only upper limits for the rarer ${ }^{57} \mathrm{Fe}$ and ${ }^{58} \mathrm{Fe}$. Similarly, there are presently only very limited results for $\mathrm{S}, \mathrm{Ni}$, and other isotope ratios. The significance of the results summarized in Figures $3-10$ for cosmic ray origin theories is discussed in a later section. 

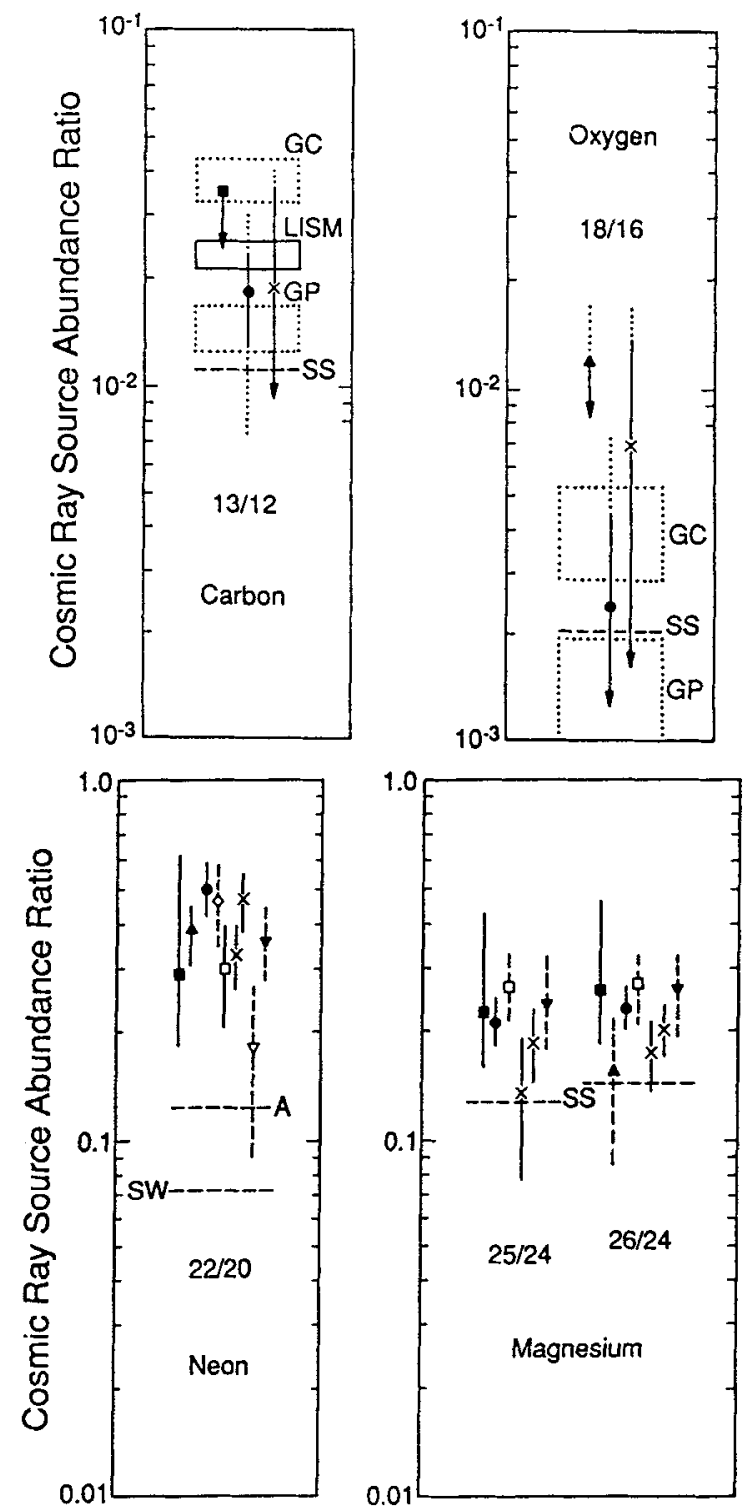

Figures 3, 4, 5, 6: Reported GCRS abundances of $\mathrm{C}, \mathrm{O}, \mathrm{Ne}$, and $\mathrm{Mg}$ are compared with the solar system (SS) composition ${ }^{50}$ (dashed lines). For $\mathrm{C}$ and $\mathrm{O}$ optical ${ }^{51}$ and millimeter wave ${ }^{52,53}$ measurements of the local interstellar medium (LISM), galactic plane (GP), and galactic center (GC) are also shown, while for Ne both the solar wind ${ }^{54}(\mathrm{SW})$ and neon- $\mathrm{A}^{55}$ compositions are indicated. The data points, ordered by energy, are based on both satellite (closed symbols) and balloon observations (open symbols), as listed in Figures 7-10. Unresolved or "mean-mass" observations are shown with dashed uncertainties. Dotted extensions to the $\mathrm{C}$ and $\mathrm{O}$ uncertainties indicate the effect of cross section uncertainties ${ }^{77}$. 

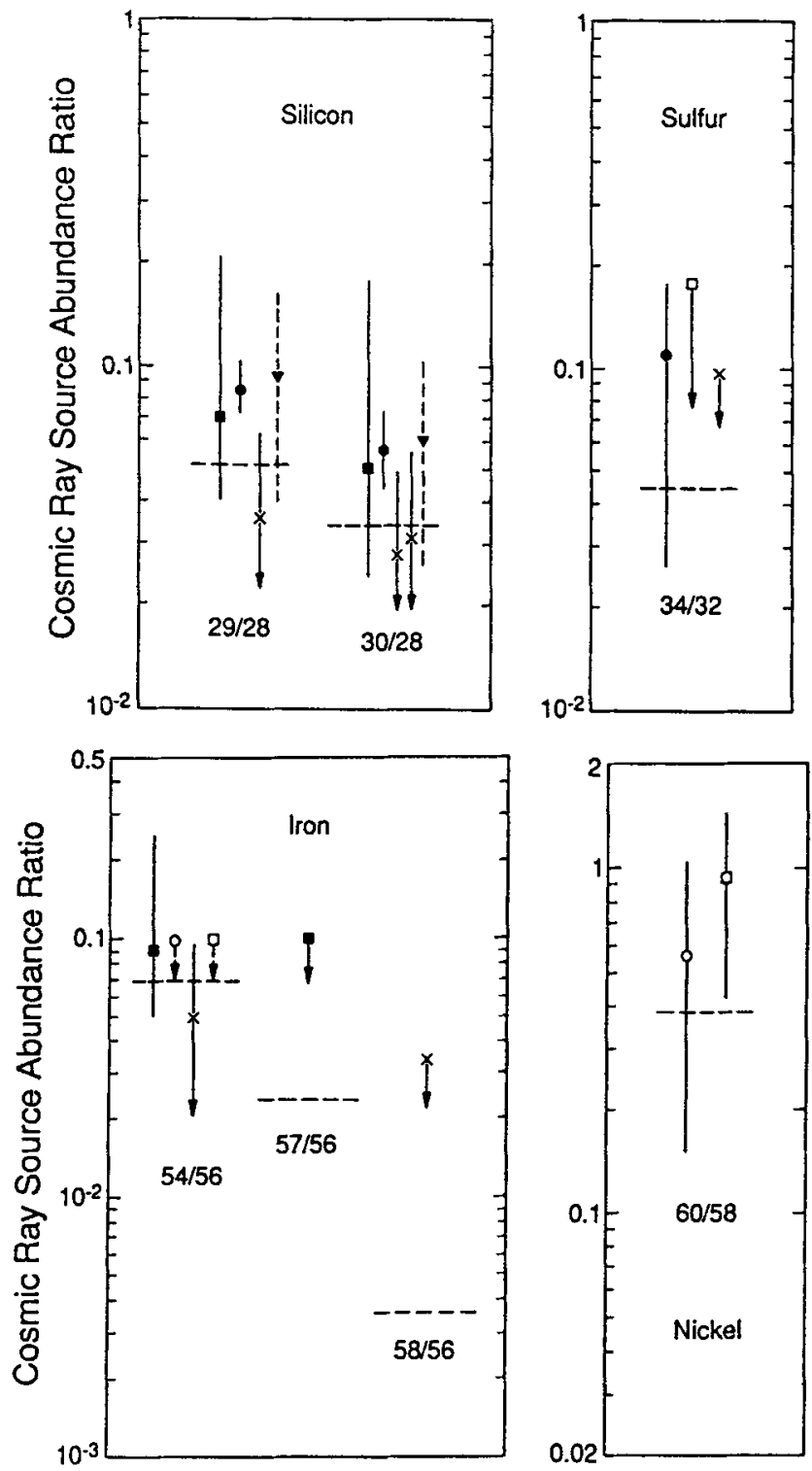

Figures 7, 8, 9 and 10: Reported source abundances of $\mathrm{Si}, \mathrm{S}, \mathrm{Fe}$, and $\mathrm{Ni}$ are compared with the solar system composition (horizontal dashed lines). The data are based on measurements by Berkeley ${ }^{56-60}$ (closed and open circles), Caltech ${ }^{61-63}$ (closed square), Chicago ${ }^{64-68}$ (closed upward triangle and open downward triangle), Goddard $^{69-70}$ (diamond), HEAO ${ }^{71-72}$ (downward triangle), Minnesota ${ }^{73-74}$ (open square), and New Hampshire $46,47,75,76$ (cross). Additional measurements and discussion can be found in reviews by Mewaldt ${ }^{78,79}$, Simpson ${ }^{80}$, Wiedenbeck ${ }^{58}$, and Meyer $^{81}$. See also the caption to Figure 3-6. 


\section{THE NITROGEN ABUNDANCE IN COSMIC RAY SOURCES}

The abundance of nitrogen in cosmic ray sources has been a controversial question over the past few years ${ }^{66,86,87,78,88,81,7,89}$. Low energy $(\sim 30-300$ $\mathrm{MeV} / \mathrm{nuc}$ ) isotope measurements indicate that the majority of observed nitrogen is ${ }^{15} \mathrm{~N}$ (presumed to be of secondary origin, since ${ }^{15} \mathrm{~N} /{ }^{14} \mathrm{~N} \simeq 0.004$ in the solar system), and that the resulting source abundance of ${ }^{14} \mathrm{~N}$ is only a few $\%$ of that of ${ }^{16} \mathrm{O}$, whereas ${ }^{14} \mathrm{~N} /{ }^{16} \mathrm{O} \simeq 0.12$ in solar system material ${ }^{90,63,91,88}$. There have also been higher energy isotope measurements and some measurements of the elemental $\mathrm{N} / \mathrm{O}$ abundance ratio that have indicated a somewhat higher ${ }^{14} \mathrm{~N} /{ }^{16} \mathrm{O}$ ratio $67,87,71$ not inconsistent with Meyer's adopted "local galactic" value ${ }^{92,7}$ of $\mathrm{N} / \mathrm{O}=0.10 \pm$ 0.05 . In general, it has been difficult to reconcile all the available measurements of ${ }^{15} \mathrm{~N} / \mathrm{N}, \mathrm{N} / \mathrm{O}$ and $\mathrm{B} / \mathrm{C}$ with the standard propagation model and cross-sections available up until 1985. This suggested the possibility that there might be substantial ${ }^{15} \mathrm{~N}$ in the source (see, e.g., the review by Meyer ${ }^{81}$ ), and also led to the suggestion that the $\mathrm{N}$ isotope results were evidence for cosmic ray reacceleration effects ${ }^{81,93}$. Guzik et al. ${ }^{94,83}$ have examined the reacceleration question and concluded that invoking reacceleration does not help explain the $B / C,{ }^{15} \mathrm{~N} / \mathrm{N}$, and $\mathrm{N} / \mathrm{O}$ results ${ }^{89,96}$.

Figure 11: Measured and calculated ${ }^{10} \mathrm{~B} / \mathrm{B}$ and ${ }^{15} \mathrm{~N} / \mathrm{N}$ ratios as a function of kinetic energy/nucleon (from Krombel and Wieden beck ${ }^{97}$ ). The calculated curves are fits that assume a source abundance of ${ }^{14} \mathrm{~N} /{ }^{16} \mathrm{O}=0.037$. Although the fits also assume a small ${ }^{15} \mathrm{~N}$ source abundance $\left({ }^{15} \mathrm{~N} /{ }^{18} \mathrm{O}=\right.$ 0.023) the observations are consistent with ${ }^{15} \mathrm{~N} /{ }^{16} \mathrm{O}=0.0$ or with the solar system value of ${ }^{15} \mathrm{~N} /{ }^{14} \mathrm{~N} \simeq 0.004$ when cross section and other uncertainties are taken into account. For references to the data see Krombel and Wiedenbeck.

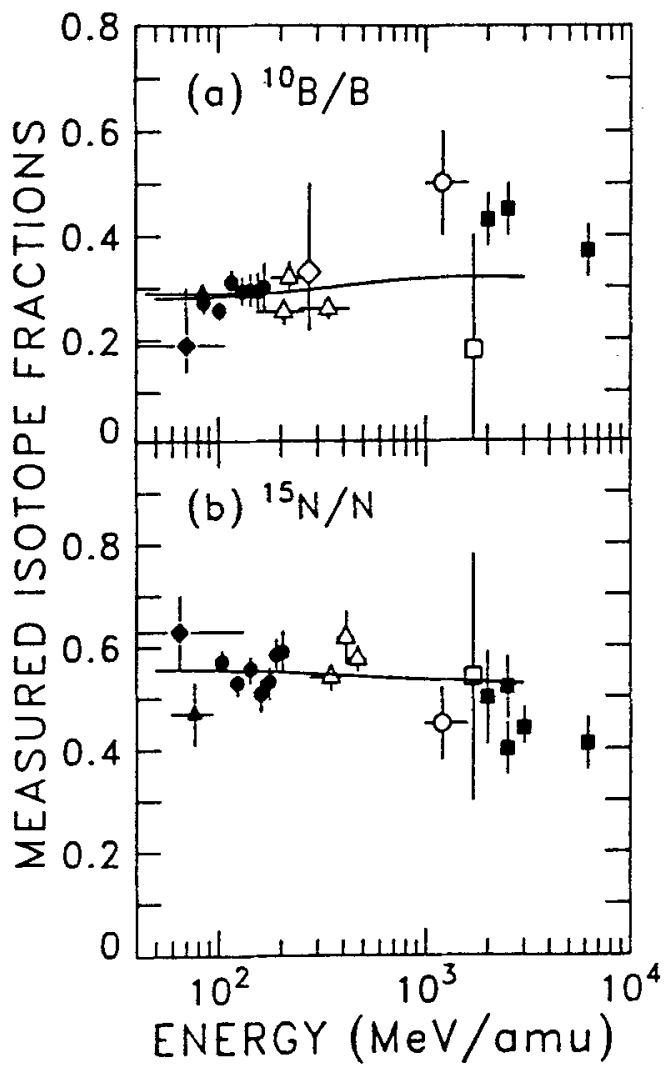


Recently, two new studies of cosmic ray nitrogen were reported that make use of newly measured cross sections. The cross section revisions result in better agreement between the observations and the propagation calculations, and both new studies confirm the low abundance of ${ }^{14} \mathrm{~N}$ in cosmic ray sources as deduced by earlier isotope studies. Thus, Krombel and Wiedenbeck ${ }^{97}$ find source abundances of ${ }^{14} \mathrm{~N} /{ }^{16} \mathrm{O}=0.037 \pm 0.017$, with ${ }^{15} \mathrm{~N} /{ }^{16} \mathrm{O} \leq 0.052$, while Webber and Gupta ${ }^{98}$ find ${ }^{14} \mathrm{~N} /{ }^{16} \mathrm{O}=0.03 \pm .01$. Neither study finds it necessary to include an excess of ${ }^{15} \mathrm{~N}$ in the source, although this possibility is not ruled out. Figure 11, from Krombel and Wiedenbeck, illustrates the agreement that has been achieved between the measurements and calculations.

Figure 12 compares the two recent determinations of the ${ }^{14} \mathrm{~N} /{ }^{16} \mathrm{O}$ ratio for cosmic ray sources with determinations of this ratio in other samples of solar system and galactic material. Note that the available measures of this ratio in solar system material are consistent with a ratio of ${ }^{14} \mathrm{~N} /{ }^{16} \mathrm{O}$ ratio $=0.125 \pm 0.01$. This includes the revised photospheric value reported at the conference by Grevesse and Anders $^{99}$, and the determinations from solar-energetic-particles ${ }^{100}$ (SEPs) which are completely independent of the photospheric values derived from spectroscopy. There is a considerable spread of $\mathrm{N} / \mathrm{O}$ values observed in the ISM, and Meyer adopts a large uncertainty for his "local galactic" value ${ }^{7}$. However,

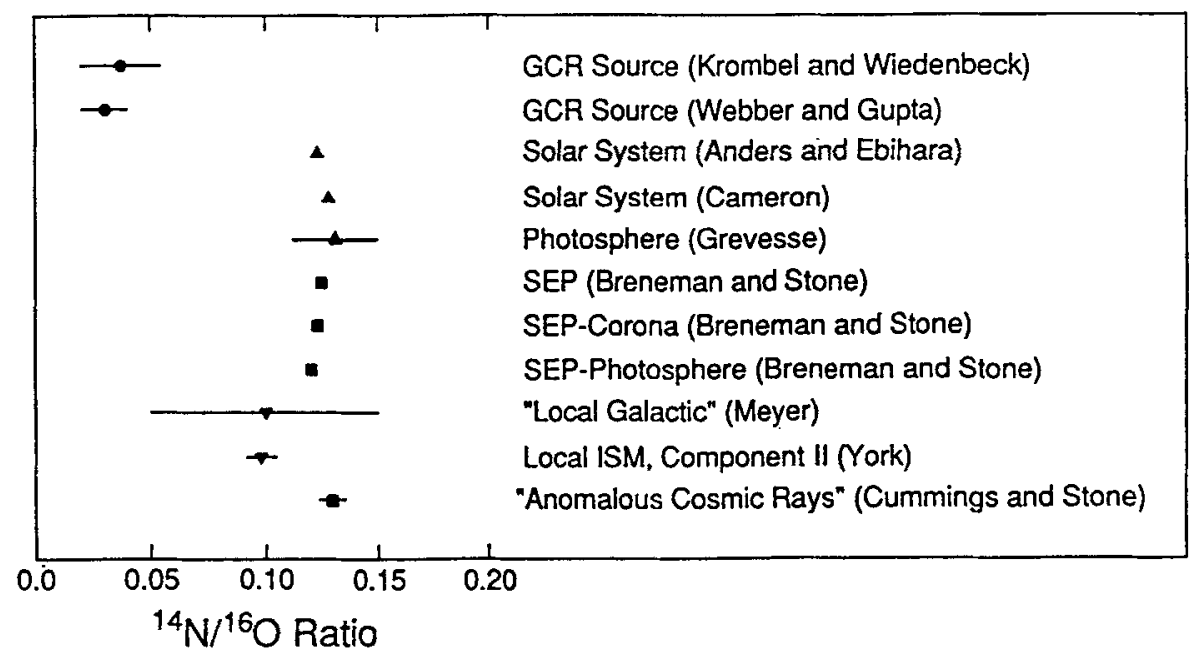

Figure 12: A summary of various determinations of the ${ }^{14} \mathrm{~N} /{ }^{16} \mathrm{O}$ ratio (adapted from Krombel and Wiedenbeck ${ }^{97}$ ), including recent measurements of cosmic ray GCRS material ${ }^{97,98}$, various solar system values based on spectroscopic ${ }^{50,82,99}$ and solar energetic particle ${ }^{100}$ (SEP) measurements, and values representative of the LISM $^{7,101}$, including the anomalous cosmic ray component ${ }^{102}$. The Cameron and the Anders and Ebihara solar system tabulations do not include uncertainties, while the size of the measurement uncertainties on the SEP-derived values is smaller than the size of the plotted points ${ }^{100}$ 
typical galactic $\mathrm{N} / \mathrm{O}$ measurements in the solar system vicinity find $\mathrm{N} / \mathrm{O} \simeq 0.10$, including York's "Component II" based on Copernicus data ${ }^{101}$. It is also interesting that the "anomalous cosmic ray" component, thought to represent a sample of the neutral ISM (see below), has a similar N/O ratio of $0.13^{102}$.

\section{INTERPRETATION OF THE COSMIC RAY SOURCE COMPOSITION}

Figure 13 and Table I present a summary of the isotopic composition of cosmic ray source material, based on a weighted mean of the results included in Figures 3 through 10 . The results in Table $I$ have been broken in to three categories in an effort to separate those confirmed characteristics that cosmic ray origin theories should address, from other less-established results and limits. The major features are the excess of neutron-rich $\mathrm{Ne}$ and $\mathrm{Mg}$ isotopes and the anomalies in the elemental abundances of CNO nuclei. In addition, of course, there is a fractionation of the elemental abundances apparently associated with first ionization potential ${ }^{6,7}$ that is not considered here. The reported excess of neutronrich Si isotopes is a noteworthy "probable" result.

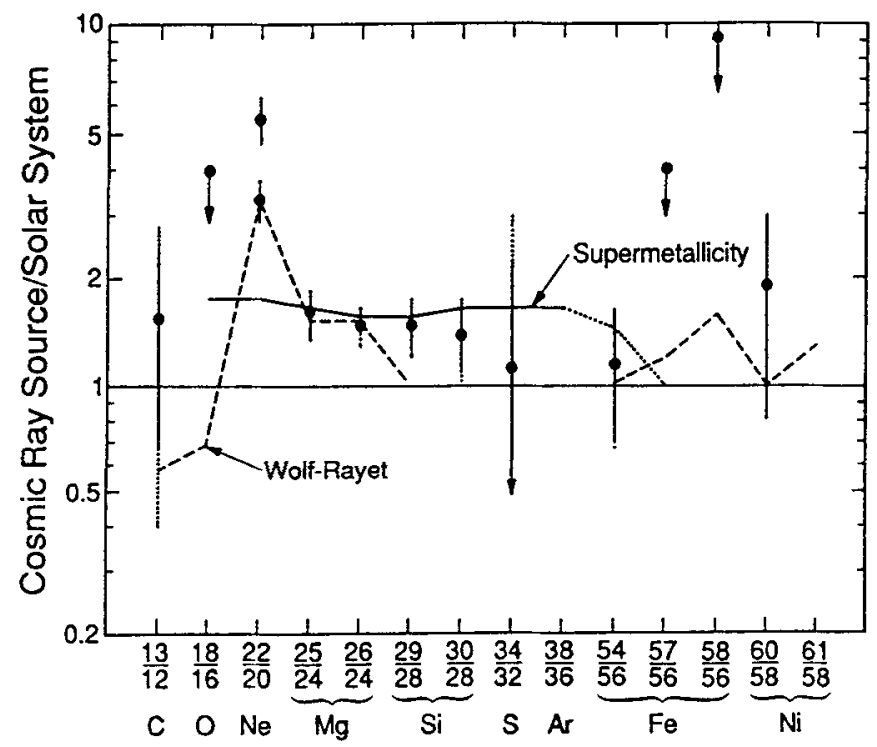

Figure 13: Comparison of measured and calculated cosmic ray source and solar system compositions. The data points are based on a weighted mean of the measurements in Figures 3-10, and Anders and Ebihara ${ }^{50}$ "solar system" values. For ${ }^{22} \mathrm{Ne} /{ }^{20} \mathrm{Ne}$ the two plotted points represent the Anders and Ebihara and the Cameron ${ }^{82}$ choices for the solar system value of this ratio. Dotted extensions to the error bars indicate the uncertainty in correcting back to the source due to cross-section uncertainties. The supermetallicity prediction for ${ }^{54} \mathrm{Fe} /{ }^{56} \mathrm{Fe}$ is shown dotted because there is some uncertainty as to whether it would depend on the initial metallicity in the same manner as the lighter isotope ratios ${ }^{95}$. 


\begin{tabular}{|c|c|c|c|c|c|}
\hline \multicolumn{2}{|c|}{ Established Results } & \multicolumn{2}{|c|}{$\begin{array}{c}\text { Probable/Possible } \\
\text { Require Confirmation }\end{array}$} & \multicolumn{2}{|c|}{ Other Limits } \\
\hline $\begin{array}{l}\text { Isotope } \\
\text { Ratio }\end{array}$ & $\begin{array}{l}\text { Cosmic Ray } \\
\text { Source } \\
\text { Solar System }\end{array}$ & $\begin{array}{l}\text { Isotope } \\
\text { Ratio }\end{array}$ & $\begin{array}{l}\text { Cosmic Ray } \\
\frac{\text { Source }}{\text { Solar System }}\end{array}$ & $\begin{array}{l}\text { Isotope } \\
\text { Ratio }\end{array}$ & $\begin{array}{l}\text { Cosmic Ray } \\
\text { Source } \\
\text { Solar System }\end{array}$ \\
\hline${ }^{22} \mathrm{Ne} /{ }^{20} \mathrm{Ne}$ & $\begin{array}{c}3.3 \pm 0.45^{\mathrm{a}} \\
\text { or } \\
5.5 \pm 0.75\end{array}$ & ${ }^{29} \mathrm{Si} /{ }^{28} \mathrm{Si}$ & $1.5 \pm 0.3$ & ${ }^{13} \mathrm{C} /{ }^{12} \mathrm{C}$ & $1.55 \pm 1.25$ \\
\hline${ }^{25} \mathrm{Mg} /{ }^{24} \mathrm{Mg}$ & $1.6 \pm 0.25$ & ${ }^{30} \mathrm{Si} /{ }^{28} \mathrm{Si}$ & $1.4 \pm 0.35$ & ${ }^{18} \mathrm{O} /{ }^{16} \mathrm{O}$ & $\leq 4$ \\
\hline${ }^{26} \mathrm{Mg} /{ }^{24} \mathrm{Mg}$ & $1.5 \pm 0.20$ & & & ${ }^{34} \mathrm{~S} /{ }^{32} \mathrm{~S}$ & $\leq 3$ \\
\hline${ }^{14} \mathrm{~N} /{ }^{16} \mathrm{O}$ & $0.25 \pm .10$ & & & ${ }^{54} \mathrm{Fe} /{ }^{56} \mathrm{Fe}$ & $1.15 \pm 0.5$ \\
\hline \multirow[t]{3}{*}{${ }^{12} \mathrm{C} /{ }^{16} \mathrm{O}$} & $\sim 2$ & & & ${ }^{57} \mathrm{Fe} /{ }^{58} \mathrm{Fe}$ & $\leq 4$ \\
\hline & & & & ${ }^{58} \mathrm{Fe} /{ }^{56} \mathrm{Fe}$ & $\leq 10$ \\
\hline & & & & ${ }^{60} \mathrm{Ni} /{ }^{58} \mathrm{Ni}$ & $1.9 \pm 1.3$ \\
\hline
\end{tabular}

The differences in composition between GCRS and SS material imply that the nucleosynthesis of these two samples of matter has differed, a discovery that has stimulated a number of new theoretical suggestions as to how such differences might have occurred. Of these, the most quantitative are the so-called "supermetallicity" model of Woosley and Weaver ${ }^{103}$, and the Wolf-Rayet model proposed by Casse and Paul ${ }^{104}$ and others ${ }^{105-108,7}$. For a discussion of these and other proposed models see Casse ${ }^{109}$ and Arnould ${ }^{131}$.

Following earlier work by Arnett ${ }^{110}$ and others, Woosley and Weaver ${ }^{103}$ pointed out that the production of neutron-rich isotopes in massive stars is proportional to the initial "metallicity" (the fraction of $\mathrm{Z}>2$ elements) of the material from which the star formed. This connection results because following $\mathrm{H}$ burning, in which the initial CNO nuclei are burned to ${ }^{14} \mathrm{~N}$, helium-burning turns ${ }^{14} \mathrm{~N}$ in to ${ }^{22} \mathrm{Ne}$ (a neutron rich nucleus) via the following series of reactions:

$$
{ }^{14} \mathrm{~N}(\alpha, \gamma){ }^{18} \mathrm{~F}\left(\beta^{+} \nu\right)^{18} \mathrm{O}(\alpha, \gamma)^{22} \mathrm{Ne}
$$

Woosley and Weaver proposed that the observed excess of neutron-rich isotopes might result if cosmic rays originate in regions of the galaxy that are metal-rich compared to the solar system. Such metal-rich regions might result in part from evolutionary effects, if cosmic rays indeed represent a younger sample of material 
than that which condensed to form the solar system, or, more likely, they might be the result of inhomogeneities in the galactic metal distribution. Woosley and Weaver predicted enhancements for a number of other neutron-rich nuclei, including ${ }^{18} \mathrm{O},{ }^{34} \mathrm{~S},{ }^{38} \mathrm{~A}$, and ${ }^{54} \mathrm{Fe}$ (see Figure 13), assuming "normal" abundances for the more numerous "alpha-particle" nuclei such as ${ }^{16} \mathrm{O}$. When normalized to ${ }^{25} \mathrm{Mg}$ and ${ }^{26} \mathrm{Mg}$, the supermetallicity model is consistent with the approximately equal enhancements observed for the $\mathrm{Mg}$ and $\mathrm{Si}$ isotopes, but it would apparently require an additional source of ${ }^{22} \mathrm{Ne}$ to explain the large ${ }^{22} \mathrm{Ne} /{ }^{20} \mathrm{Ne}$ ratio in cosmic rays.

In the Wolf-Rayet (WR) model it is proposed that a fraction of heavy cosmic rays originate from material expelled by Wolf-Rayet stars ${ }^{104}$. These massive stars are undergoing significant mass loss $\left(\sim 10^{-5}\right.$ solar masses per year) by means of high-velocity stellar winds (several thousand $\mathrm{km} / \mathrm{sec}$ ). As a result they have been stripped of their hydrogen envelopes, and helium-burning products including ${ }^{12} \mathrm{C}$, ${ }^{16} \mathrm{O}$, and ${ }^{22} \mathrm{Ne}$ have been exposed and are being expelled from their surface. The high-velocity stellar winds also make WR stars attractive sites for the acceleration of cosmic rays to modest energies ${ }^{104}$, where they might be further accelerated by supernova shock waves. Figure 14, adapted from the work of Prantzos et al. ${ }^{111}$, shows the mass fraction of representative isotopes that reach the surface of a 60 solar mass WR star as a function of time. Note that when the helium-burning products reach the surface, and the star enters the WC and WO phases, there are large enhancements of ${ }^{22} \mathrm{Ne},{ }^{12} \mathrm{C}$, and ${ }^{16} \mathrm{O}$, and somewhat smaller enhancements of ${ }^{25} \mathrm{Mg}$ and ${ }^{26} \mathrm{Mg}$ in the material that gets ejected. The enhancement of the neutron-rich $\mathrm{Si}$ isotopes on the other hand, is much smaller.

Figure 14: Calculations of the mass fraction $\left(\mathrm{X}_{\mathrm{s}}\right)$ of various isotopes that reach the surface of a 60 mass WR star as a function of time, adapted from similar figures by Prantzos et al. ${ }^{111}$. Note the break in the time scale at $5 \times 10^{6}$ years.

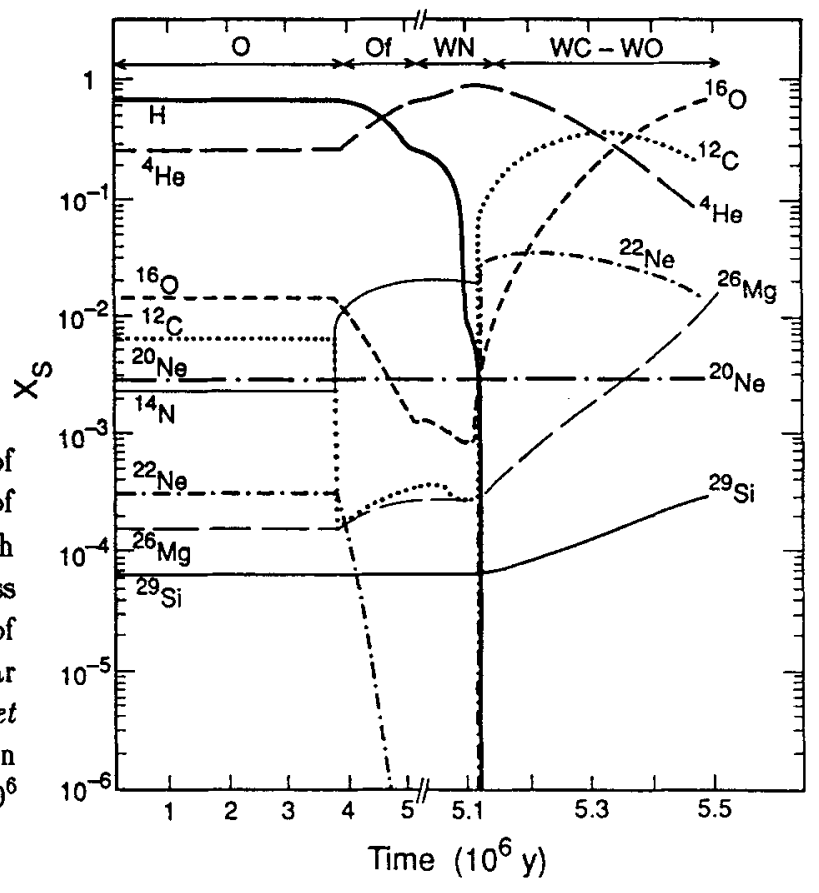


Casse and Paul proposed that a fraction of cosmic rays originate from WR material, with the bulk of cosmic rays originating out of so-called "normal" material. Thus, for example, to agree with the observed ${ }^{22} \mathrm{Ne}$ overabundance in GCRS material the large (factor of $\sim 100$ ) enhancement of ${ }^{22} \mathrm{Ne}$ in WR material must be diluted. The predicted enhancements for other isotopes are then correspondingly smaller. Casse and $\mathrm{Paul}$ also suggested (see also Meyer ${ }^{105}$ ) that this scenario could explain the fact that $\mathrm{C} / \mathrm{O} \simeq 1$ in cosmic rays, compared to $\mathrm{C} / \mathrm{O}$ $\simeq 0.5$ in the solar system. Although they did not specify the origin of the "normal" material, Meyer ${ }^{7}$ has proposed that the bulk of cosmic rays were extracted from the coronae of ordinary $F$ to $M$ stars, which would explain the observed similarity of the GCRS and solar energetic particle elemental compositions.

Figure 13 includes predictions for the WR model by Prantzos et al. ${ }^{108}$, normalized to the observed ${ }^{25} \mathrm{Mg}$ and ${ }^{26} \mathrm{Mg}$ enhancements. Note that in this case the model agrees with the observed ${ }^{22} \mathrm{Ne}$ abundance but it does not produce an excess of either ${ }^{29} \mathrm{Si}$ and ${ }^{30} \mathrm{Si}$; one of several differences from the supermetallicity model. The WR model also predicts enhancements of s-process nuclei such as ${ }^{58} \mathrm{Fe}$, and it leads to depressed ${ }^{13} \mathrm{C} /{ }^{12} \mathrm{C}$ and ${ }^{18} \mathrm{O} /{ }^{16} \mathrm{O}$ ratios as a result of the large amount of pure ${ }^{12} \mathrm{C}$ and ${ }^{16} \mathrm{O}$ expected from WR stars.

The WR model does lead to rather remarkable agreement with many existing cosmic ray observations, but there are some potential problems with the model, including the depletion of ${ }^{14} \mathrm{~N}$ in GCRS material, and the fact that the model apparently requires (if it is to explain the cosmic ray $\mathrm{C} / \mathrm{O}$ ratio) that about $25 \%$ of all cosmic rays heavier than He result from material processed by WR stars. In addition, Meyer ${ }^{89}$ has pointed out that in order to fit simultaneously the observed $\mathrm{Ne}$ and $\mathrm{Mg}$ isotope ratios without invoking additional sources or assumptions, it is apparently necessary that first ionization potential effects (or other atomic effects) fractionate WR material in the same way as the bulk of cosmic ray material, which he found difficult to envision in the context of this model.

There is one additional model that bears mention because it introduces an alternative point of view. Olive and Schramm ${ }^{112}$ have proposed that supernova explosions occurring at the time of formation of the solar system may have added an excess of $\alpha$-particle nuclei (e.g., ${ }^{12} \mathrm{C},{ }^{16} \mathrm{O}$, and ${ }^{20} \mathrm{Ne}$, etc) and r-process material to the proto-solar nebula, as well as material that led to isotopic anomalies in meteorites. In this model cosmic rays could be representative of the interstellar medium (and only appear to be enriched in neutron-rich isotopes), while the solar system, used as our standard, is anomalous because of the addition of material just prior to its formation. Tests of this "anomalous solar system" model include predicted enhancements in cosmic rays of s-process nuclei (for which there is currentiy no evidence among ultra-heavy $(Z \geq 30)$ cosmic ray nuclei ${ }^{5}$ ) and a (factor of $\sim 2$ ) enhancement of ${ }^{13} \mathrm{C}$ in cosmic rays. If this model is correct, cosmic ray measurements might shed light on the events associated with the formation of the solar system.

With a few exceptions, the models designed to explain the observed isotopic composition of GCRS material have not addressed the depletion of ${ }^{14} \mathrm{~N}$ in this 
material. The WR model would not be expected to result in a depletion of ${ }^{14} \mathrm{~N}$, and might result in a small ${ }^{14} \mathrm{~N}$ excess ${ }^{89}$, since WR stars do eject considerable $\mathrm{N}$ over their lifetime (especially during their WN phase; see Figure 14). Similarly, with respect to the supermetallicity model, one would expect the $\mathrm{N}$ abundance to generally increase with the metallicity rather than decrease, as $\mathrm{N}$ is a "secondary" product of nucleosynthesis. Audouze ${ }^{113}$ has discussed several possibilities of obtaining a low $\mathrm{N}$ abundance in cosmic rays. Both he, and more recently Webber and Gupta ${ }^{98}$ have pointed out that the ${ }^{14} \mathrm{~N}$ depletion is numerically equal to the enhancement of ${ }^{22} \mathrm{Ne}+{ }^{25} \mathrm{Mg}+{ }^{26} \mathrm{Mg}$ in GCRS material. They suggested that this might be significant since ${ }^{14} \mathrm{~N}$ is burned to form these heavier neutron-rich isotopes (particularly ${ }^{22} \mathrm{Ne}$ ) during helium-burning, but did not suggest an astrophysical scenario where this numerical equality would apply (see also the discussion by Meyer ${ }^{89}$ ). Some years ago Hainebach et al. ${ }^{85}$ and Silberberg et al. ${ }^{115}$ suggested that a low nitrogen abundance could result if the bulk of cosmic rays originate from supernova ejecta; perhaps such models should now be re-examined.

The abundance of isotopes such as ${ }^{13} \mathrm{C}$ and ${ }^{18} \mathrm{O}$ in cosmic ray sources is also an important test of galactic evolution models. As indicated in Figure 3, there is good evidence from optical and millimeter-wave measurements for a ${ }^{13} \mathrm{C} /{ }^{12} \mathrm{C}$ ratio in the local neighborhood which is a factor of 2 greater than the solar system value, and similar differences have been obtained for ${ }^{18} \mathrm{O} /{ }^{16} \mathrm{O}$ and other isotope ratios $^{52,53}$. The ${ }^{13} \mathrm{C} /{ }^{12} \mathrm{C}$ observations in particular, which are consistent with some galactic chemical evolution models ${ }^{116,117}$, provide direct evidence that the composition of the local neighborhood has evolved since the formation of the Sun. The present GCR source abundance of ${ }^{13} \mathrm{C}$ has a large uncertainty, due in part to uncertainties in the cross sections required to correct for the sizable secondary contribution of ${ }^{13} \mathrm{C}$ produced during cosmic ray transport ${ }^{56,125}$. Recent (but as yet unreported) cross-section measurements, combined with improved cosmic ray measurements, should make it possible to differentiate between the Wolf-Rayet, solar system, and local galactic values.

At present, it is perhaps fair to say that while none of the above-mentioned models is completely consistent with all of the observations (see Figure 13), there are still a number of important isotope abundance ratios that need to be measured to completely test these models, and it is of course possible that new or revised models will be required. As an example, a possible extension of the WR model might be to dilute WR material with material characteristic of the present-day interstellar medium (rather than solar-system material) in an attempt to account for evolutionary effects over the $4.5 \times 10^{9}$ years since the formation of the solar system $^{79}$.

\section{COSMIC RAY ACCELERATION AND REACCELERATION}

Supernova explosions in the galaxy are generally believed to be the source of the energy responsible for accelerating cosmic ray nuclei to high energy. It is not known, however, whether this acceleration occurs immediately via shock acceleration during the birth and infancy of the supernova, or much later on, as 
supernova shock waves from older supernovae make multiple encounters with material in the interstellar medium. In one case cosmic. rays would consist of freshly synthesized material ejected from recent supernovae, while in the second cosmic rays would include contributions from element synthesis integrated over the age of the galaxy.

This fundamental question could be addressed with future cosmic ray isotope measurements by measuring radioactive isotopes produced during the supernova explosion that decay only by electron capture, including ${ }^{56} \mathrm{Ni},{ }^{57} \mathrm{Co}$, and ${ }^{59} \mathrm{Ni}$, which have radioactive half-lives ranging from a few days $\left({ }^{56} \mathrm{Ni}\right)$ to $\sim 10^{5}$ years $\left({ }^{59} \mathrm{Ni}\right.$ ) (see Soutoul et al. $\left.{ }^{119}\right)$. Once accelerated and stripped of their electrons these nuclei can no longer decay, and their relative abundances therefore preserve a record of the time-delay between nucleosynthesis and acceleration to high energies.

When the delay exceeds a few days, most of the ${ }^{56} \mathrm{Ni}$ will have decayed to ${ }^{56} \mathrm{Co}$, which subsequently decays to ${ }^{56} \mathrm{Fe}$, producing the 0.847 and $1.238 \mathrm{MeV} \gamma$ rays recently observed from $\mathrm{SN} 1987 \mathrm{~A}$ in the LMC. If the time delay is much greater than a thousand days, most of the ${ }^{57} \mathrm{Co}$ (produced during the first few days following the explosion by the decay of ${ }^{57} \mathrm{Ni}$ ) will have decayed to ${ }^{57} \mathrm{Fe}$. Finally, if the time delay is much more than $\sim 10^{5}$ years, the ${ }^{59} \mathrm{Ni}$ will have decayed to ${ }^{59} \mathrm{Co}$. On the basis of isotope measurements to date, it is possible to say only that the delay is $>30$ days, based on the fact that the dominant isotope of $\mathrm{Fe}$ in cosmic rays is ${ }^{56} \mathrm{Fe}$, as it is in the solar system, and that ${ }^{56} \mathrm{Fe}$ is more abundant than ${ }^{56} \mathrm{Ni}$. Although attempts have been made to address this problem using element abundance ratios such as $\mathrm{Co} / \mathrm{Ni}^{120}$, this approach involves additional assumptions, and is subject to cross section uncertainties ${ }^{121}$. However, future measurements of $\mathrm{Fe}, \mathrm{Ni}$, and $\mathrm{Co}$ isotopes should provide critical information about supernovae and their role in accelerating cosmic rays.

During the past decade it has been demonstrated that supernova shock waves traversing interstellar space provide an attractive means of accelerating galactic cosmic rays (see, e.g., Blandford and Ostriker ${ }^{122}$, and the reviews by Axford ${ }^{123}$ and Cesarsky ${ }^{96}$ ). In particular, such shock waves appear to satisfy the energetic requirements for cosmic ray acceleration and they are capable of producing the observed energy spectra. If cosmic rays are indeed undergoing sporadic "reacceleration" in the interstellar medium (possibly after being injected by an initial acceleration event) we would expect the mean Lorentz factor over the lifetime of cosmic rays to be less than that determined by the arriving energy, which may mean that the present data are compatible with a shorter cosmic ray "age" than $\sim 10^{7}$ years $^{126}$. In addition, the energy dependence of secondary/primary isotope ratios such as ${ }^{2} \mathrm{H} /{ }^{4} \mathrm{He}$ and ${ }^{3} \mathrm{He} /{ }^{4} \mathrm{He}$ can test the significance of reacceleration or other energy changing processes. Although the effects of reacceleration on the abundances of secondary (especially radioactive) isotopes are still controversial ${ }^{126-128}$, it is clear that the predicted energy dependence of ratios such as those in Figures 1 and 2 would be altered if reacceleration effects are indeed significant. 


\section{ISOTOPIC COMPOSITION OF THE ANOMALOUS COSMIC RAYS}

Observations of quiet-time cosmic rays below $\sim 50 \mathrm{MeV} /$ nucleon may offer a unique opportunity to study an additional sample of matter from interstellar space. During the 1972-1978 solar minimum period, measurements revealed anomalous enhancements in the low-energy spectra of the elements $\mathrm{O}, \mathrm{N} \mathrm{He}$, and $\mathrm{Ne}$ relative to those of other elements such as B and C (see e.g., the review by Gloeckler ${ }^{129}$ and the paper at this conference by Webber ${ }^{130}$ ). Recent Voyager observations ${ }^{102}$ have also revealed similar enhancements in the spectra of $\mathrm{H}, \mathrm{C}$, and Ar. Following a suggestion by Fisk et al. ${ }^{132}$, this so-called "anomalous" cosmic ray (ACR) component is now generally believed to represent interstellar neutral particles that have drifted in to the heliosphere, become ionized by the solar wind or UV radiation, and then accelerated to energies $>10 \mathrm{MeV} /$ nucleon, probably at the solar wind termination shock ${ }^{133}$. A unique prediction of this model, for which there is indirect evidence, is that the ACR component should be singly ionized. If the model of Fisk et $a l$. is indeed correct, then the ACR component represents a direct sample of the local interstellar medium that carries important information about galactic evolution in the solar neighborhood over the time interval since the formation of the solar nebula ${ }^{134}$. Indeed, Cummings and Stone ${ }^{102}$ have already demonstrated how the elemental abundances of the ACR component can be used to measure the neutral composition of the LISM.

Although there is only very limited information available on the isotopic composition of ACR nuclei, Figure 15 compares presently available results for $\mathrm{He}, \mathrm{N}$, $\mathrm{O}$, and $\mathrm{Ne}$ with calculated curves based on a two component description of lowenergy cosmic rays ${ }^{134}$. At energies below $\sim 50 \mathrm{MeV} /$ nucleon, the GCR component, assumed to have the same isotopic composition as is measured at higher energies, is mixed with an ACR component of various assumed isotopic compositions (as indicated in Figure 15), taking into account the intensity of these two components as a function of energy/nucleon. Note that for ${ }^{3} \mathrm{He}$ and ${ }^{15} \mathrm{~N}$, two isotopes that are dominated by secondary production at higher energies ( $>100 \mathrm{MeV} /$ nucleon), there is a sharp drop in the relative abundance of ${ }^{3} \mathrm{He}$ and ${ }^{15} \mathrm{~N}$ at just the energy where the elemental composition changes, as a result of the fact that the ACR component contains essentially pure ${ }^{4} \mathrm{He}$ and ${ }^{14} \mathrm{~N}$, uncontaminated by secondaries as are galactic cosmic rays. A similar decrease would be expected for ${ }^{18} \mathrm{O} /{ }^{16} \mathrm{O}$, but the present data are not clear on this point.

For ${ }^{22} \mathrm{Ne} /{ }^{20} \mathrm{Ne}$ the available data also suggest a lower ratio than is observed for higher energy ( $>100 \mathrm{MeV} /$ nucleon) galactic cosmic rays, and they apparently also favor a lower ${ }^{22} \mathrm{Ne} /{ }^{20} \mathrm{Ne}$ ratio than has been deduced for the cosmic ray source composition $\left({ }^{22} \mathrm{Ne} /{ }^{20} \mathrm{Ne}=0.43\right.$; labeled CRS). If confirmed by later measurements, this difference would be important because it would imply that the nucleosynthesis of the GCR and ACR components has differed, arguing against models which suggest that a majority, if not all, cosmic rays represent a sample of ISM material. Although the large uncertainties on the presently available data (all of which result from the 1972-78 solar minimum) do not allow any definitive conclusions to be drawn about the composition of the ISM at this time, they do illustrate the 
potential of such studies. While it is unfortunate that there were no high-resolution isotope spectrometers in space during the 1987 solar minimum that could improve on these low energy measurements, instruments proposed for future missions can provide a factor of 10 to 100 improvement in collecting power in this low energy region.
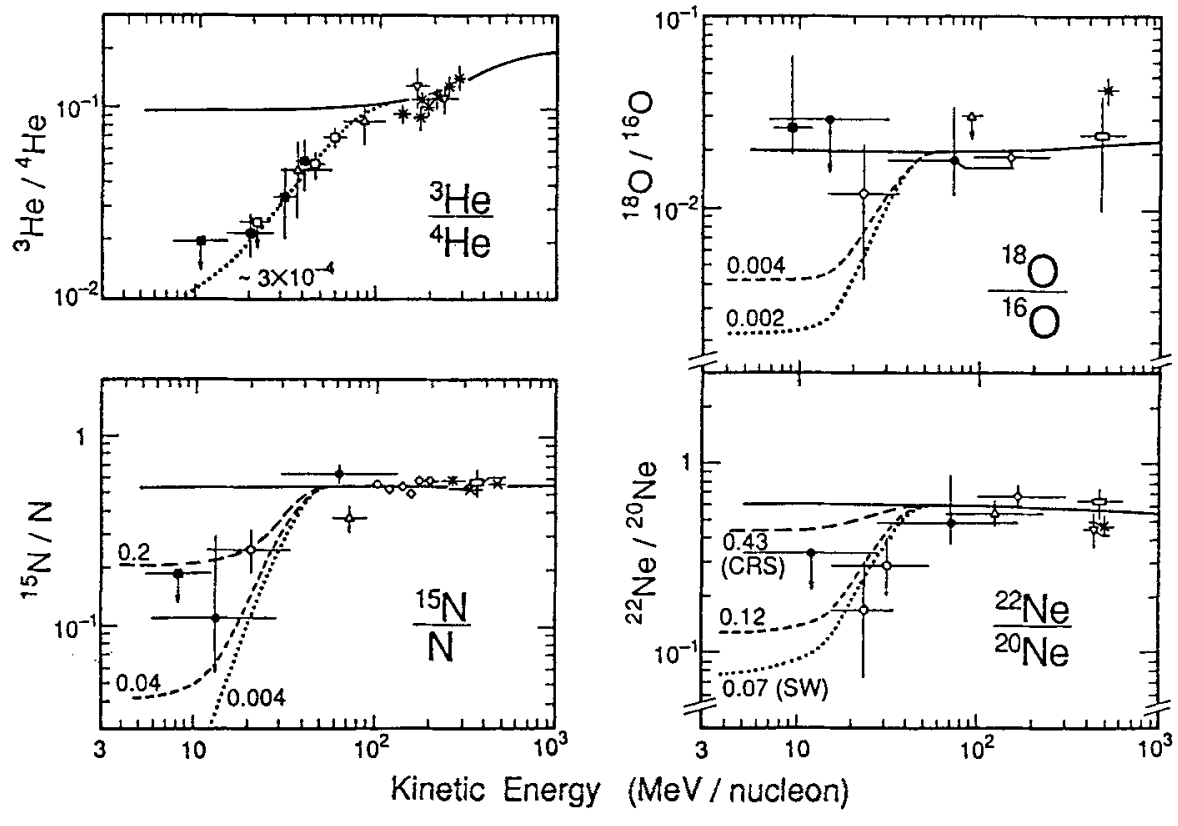

Figure 15: Measured and calculated cosmic ray isotope abundance ratios. At energies below $\sim 50 \mathrm{MeV} /$ nucleon the calculated ratios assume various compositions for the ACR component as indicated by the labeled dashed and dotted lines. The calculated GCR ratios at energies $>30 \mathrm{MeV} /$ nucleon $^{124}$ have been extrapolated to lower energies to indicate the expected composition if the ACR component were absent. The measurements are from groups at Berkeley ${ }^{56,57,91}$ (diamond), Caltech $^{61,63,134,135}$ (filled circle and square), Chicago ${ }^{64,66,114}$ (triangle), Goddard ${ }^{42,69}$ (rectangle), Maryland ${ }^{27}$ (inverted triangle), Minnesota ${ }^{73}$ (star), the University of New Hampshire ${ }^{18,47,88,136}$ (cross), Pioneer $-10^{25}$ (open square), and Voyager ${ }^{137}$ (open circle).

\section{FUTURE PROSPECTS}

Our knowledge of the isotopic composition of cosmic ray source material is still very limited. Only the $\mathrm{Ne}, \mathrm{Mg}$, and $\mathrm{Si}$ isotopes have had their source abundance determined to an accuracy of $\sim 30 \%$ or better, and in each case differences from the solar system composition have been found. If such observations are to be extended to other elements such as $\mathrm{Fe}$ and $\mathrm{Ni}$, and if we are to read the radioactive clocks that record the time-scales of cosmic ray acceleration and transport, it will be necessary to expose larger instruments in space. The 
techniques for resolving isotopes have now been proven, but they need to be applied on a larger scale.

Over the next few years we can expect a factor of $\sim 10$ improvement on the collecting power of the ISEE-3 instruments as a result of instruments that will fly on the Ulysses, CRRES and ISTP/WIND missions. These experiments will determine the relative composition of the more abundant isotopes at energies below $\sim 300 \mathrm{MeV} /$ nucleon. At somewhat higher energies, further results can be expected from balloon-borne experiments. Beyond this, there are two major proposed projects that would improve on existing observations by more than two orders of magnitude: the Advanced Composition Explorer (ACE), and Astromag, a superconducting magnet facility for particle astrophysics on the Space Station.

The Advanced Composition Explorer (ACE) would measure the isotopic and elemental composition of several samples of matter with unprecedented resolution and collecting power, including galactic cosmic rays $(30-400 \mathrm{MeV} / \mathrm{nucleon})$, the "anomalous" cosmic ray component, energetic particles accelerated in solar flares over two decades in energy/nucleon, and the solar wind. Thus ACE will measure and compare the elemental and isotopic composition of several samples of matter of differing origins. ACE is one of four possible new Explorer missions that was recently selected for a Phase-A study under the Explorer Concept Study program.

Astromag ${ }^{138,139}$, which was included as a possible facility in the recent Announcement of Opportunity for Space Station Attached Payloads, offers the possibility to extend cosmic ray isotope measurements to energies from $\sim 2$ to possibly $>10 \mathrm{GeV} /$ nucleon, allowing clocks such as ${ }^{10} \mathrm{Be}$ to be read over a wide range of time-dilation factors, and extending measurements of the source composition up to high energies ${ }^{140}$. Astromag would also measure the spectra of antiprotons, electrons, positrons, and heavy nuclei, and make a sensitive search for antinuclei.

Figure 16: Comparison of the collecting power of previous (ISEE-3, CRIE), planned (EHIC, Ulysses, CRRES, ISTP/WIND), and proposed (ACE, Astromag) cosmic ray isotope spectrometers, in addition to a typical balloon instrument.

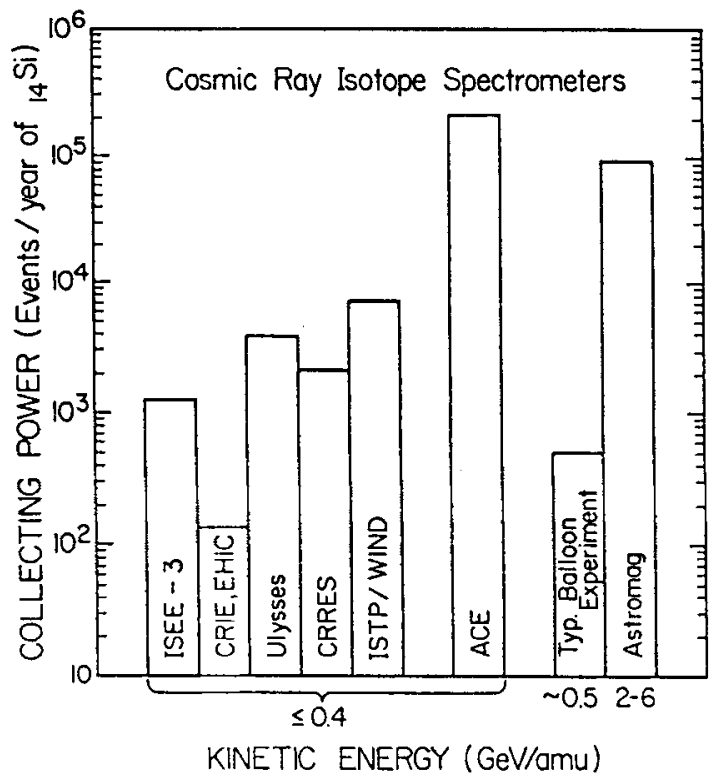


Figure 16 compares the collecting power of past, planned and proposed isotope experiments. In comparing instruments of comparable mass resolution and background rejection characteristics, it is the collecting power of the instrument that determines the accuracy of the results. As indicated in Figure 16, the collecting power of both ACE and Astromag would be more than an order of magnitude greater than that of any previous or planned experiment, sufficient to obtain definitive measurements of even rare species. Thus, on this anniversary of the discovery of heavy elements in cosmic rays we may be on the threshold of obtaining greatly improved knowledge of the isotopic composition of these elements if new instruments such as those described above can be launched in to space.

Acknowledgements: I am grateful to the organizers of this conference for their kind hospitality and to T. L. Garrard, E. C. Stone, W. R. Webber, J. P. Wefel, M. E. Wiedenbeck, and S. E. Woosley for helpful discussions. This work was supported in part by NASA under grants NAG5-722 and NGR 05-002-160.

\section{REFERENCES}

1)Freier, P. S., E. J. Lofgren, E. P. Ney, F. Oppenheimer, H. L. Bradt and B. Peters, Phys. Rev. 74, 213 (1948).

2)Freier, P. S., E. J. Lofgren, E. P. Ney and F. Oppenheimer, Phyo. Rev. 74, 1818 (1948).

3) Bradt, H. L., and B. Peters, Phyo. Rev. 74, 1828 (1948).

4)Lund, N., Symposizm on Cosmic Abundances of Matter (Minneapolis), AIP Conf. Proc. (1988).

5) Binns, W. R., T. L. Garrard, M. H. Israel, J. Klarmann, E. C. Stone, and C. J. Waddington, op. cit. (1988).

6) Casse, M., and P. Goret, Ap. J. 221, 703 (1978).

7) Meyer, J. P., Ap. J. Suppl. 57, 173 (1985).

8) Ormes, J. F. and R. J. Protheroe, Ap. J. 272, 756 (1983).

9) Garcia-Munoz, M., J. A. Simpson, T. G. Guzik, J. P. Wefel and S. H. Margolis, Ap. J. 04,269 (1987).

10) Golden, R. L., S. Horan, B. G. Mauger, G. D. Badhwar, J. L. Lacy, S. A. Stephens, R. R. Daniel and J. E. Zipse, Phy. Rev. Lettero 43, 1196 (1979).

11) Bogomolov, E. A., N. D. Lubyanays, V. A. Romanov, S. V. Stepanov and M. S. Shulakove, Proc. 16th Intemat. Cosmic Ray Conf. (Kyoto) 1, 330 (1979).

12) Buffington, A., C. D. Orth, and G. F. Smoot, Ap. J. 199, 898 (1975).

13) Muller, D. and J. Tang, Ap. J. 312, 183 (1887).

14) Golden, R. L., B. G. Mauger, S. Horan, G. D. Badhwar, J. L. Lacy, J. E. Zipse, R. R. Daniel, and S. A. Stephans, Proc. 19th Intemat. Cosmic Ray Conf. (La Jolla) 2, 374 (1985).

15) Engelmann, J. J., P. Goret, E. Juliusson, L. Koch-Mirsmond, N. Lund, P. Masse, I. L. Rasmussen, and A. Soutoul, Astron. Astrophys. 74, 12 (1985).

16) Mewaldt, R. A., Ap. J. 311, 979 (1986).

17) Soutoul, A., J. J. Engelmann, P. Ferrando, L. Koch-Miramond, P. Masse and W. R. Webber, Proc. 19th Internat. Coomic Ray Conf. (La Jolla) 2, 8 (1985).

18) Webber, W. R. and S. M. Yushak, Ap. J. 275, 391 (1983).

19) Webber, W. R., R. L. Golden and R. A. Mewaldt, Ap. J. 312, 178 (1887). 
20) Meyer, J. P., Thesis, Universite d'Paris (1974).

21) Cowsik, R. and T. K. Gaisser, Proc. 17th Internat. Cosmic Ray Conf. (Paris) 2, 218 (1981).

22) Jordan, S. P., Ap.J. 201, 207 (1985).

23) Kroeger, R., Ap. J. 303, 816 (1986).

24) Garcia-Munoz, M., G. M. Mason and J. A. Simpson, Ap. J. 202, 265 (1975).

25) Teegarden B. J., T. T. von Rosenvinge, F. B. McDonald, J. H. Trainor and W. R. Webber, Ap. J. 202, 815 (1975).

26) Beatty, James J., Ap. J. 311, 425 (1986).

27) Leech, H. W. and J. J. O'Gallagher, Ap.J. 221, 1110 (1978).

28) Webber, W.R. and N. J. Schofield, Proc. 14th Internat. Cosmic Ray Conf. (Munich) 1, 312 (1975).

29) Bogomolov, E. A., G. I. Vasilyev, S. Yu. Krut'kov, N. D. Lubyanaya, V. A. Romanov, S. V. Stepanov, and M. S. Shulakova, Proc. 2oth Internat. Cosmic Ray Conf. (Moscow) 2, 65 (1987).

30) Webber, W. R., Proc. 20th Internat. Cosmic Ray Conf. (Moscow) 8, 65 (1987).

31) Stephens, S. A., Nature 289, 267 (1981).

32) Stephens, S. A., Proc. 17th Internat. Cosmic Ray Conf. (Paris) 99, 199 (1981).

33) Ginzburg, V. L. and V. S. Ptuskin, Soviet Astr. Letters 7, 325 (1981).

34) Lagage, P. O. and C. J. Cesarsky, Astron. Astrophys. 147, 127 (1985).

35) Morfill, G. E., P. Meyer and R. Lust, Ap. J. 296, 670 (1985).

36)Koch, L., J. J. Engelmann, P. Goret, E. Juliusson, N. Petrou, Y. Rio, A. Soutoul, B. Byrnak, N. Lund, B. Peters, I. L. Rasmussen, M. Rotenberg and N. Westergaard, Astron. Astrophys. 102, L9 (1981).

37) Guzik, T. G. and J. P. Wefel, private communication (1986).

38) Wiedenbeck, M. E. and D. E. Greiner, Astrophys. J. 239, L139 (1980).

39) Wiedenbeck, M. E., Proc. 18th Int. Cosmic Ray Conf. (Bangalore) 9, 147 (1983).

40) Garcia-Munoz, M., G. M. Mason and J. A. Simpson, Ap. J. 217, 859 (1977).

41) Garcia-Munoz, M., J. A. Simpson and J. P. Wefel, Proc. 17th Int. Conf. Cosmic Rays (Paris) 2, 72 (1981).

42) Hagen, F. A., A. J. Fisher and J. F. Ormes, Ap. J., 212, 262 (1977).

43) Young, J. S., P. S. Freier and C. J. Waddington, Proc. 16th Internat. Cosmic Ray Conf. (Kyoto) 1, 442, (1979).

44) Webber, W. R., J. A. Lezniak, J. C. Kish and G. A. Simpson, Ap. Letters, 18, 125, (1977).

45) Webber, W. R. and J. Kish, Proc. 16th Int. Conf. Cosmic Rays (Kyoto) 1, 389, (1979).

46) Webber, W. R., J. C. Kish and D. A. Schrier, Proc. 19th Int. Cosmic Ray Conf. (La Jolla) 2, 88, (1985).

47) Webber, W. R., Ap. J., 252, 386 (1982).

48) Simon, H., R. Scherzer, and W. Enge, Astron. Astrophys. 75, 114 (1979).

49) Guzik, T. G. and J. P. Wefel, Proc. 19th Int. Coomic Ray Conf. (La Jolla) 2, 76 (1985).

50) Anders, E. and M. Ebihara, Geochim. Cosmochim. Acta. 40, 2363 (1982).

51) Hawkins, I. and M. Jura, Ap. J. 317, 926 (1987).

52) Penzias, Arno A., Science 208, 663 (1983).

53) Wannier, P. G., Ann. Rev. Astron. Astrophys. 18, 399 (1980).

54) Geiss, J., F. Buehler, H. Cerutti, P. Eberhardt and C. Filleux, Apollo 16 Preliminary Science Report (NASA SP-315), 14-1 (1972).

55) Podosek, F. A., Ann. Rev. Astron. Astrophys. 16, 293 (1978). 
56) Wiedenbeck, M. E. and D. E. Greiner, Ap. J. 247, L119 (1981).

57) Wiedenbeck, M. E. and D. E. Greiner, Phys. Rev. Lett. 46, 682 (1981).

58) Wiedenbeck, M. E., Adv. Space Res. 4, 15 (1984).

59) Tarle, G., S. P. Ahlen and B. G. Cartwright, Ap. J. 230, 607 (1979).

60) Tarle, G., S. P. Ahlen, B. G. Cartwright and M. Solarz, Proc. 16th Int. Conf. Cosmic Rays (Kyoto) 1, 455 (1979).

61) Mewaldt, R. A., J. D. Spalding, E. C. Stone and R. E. Vogt, Ap. J. (Letters) 235, L95 (1980).

62) Op. Cit., 236, L121 (1980).

63) Op. Cit., 251, L27 (1981).

64) Garcia-Munoz, M., J. A. Simpson and J. P. Wefel, Ap. J. (Letters) 232, L95 (1979).

65) Garcia-Munoz M., J. A. Simpson and J. P. Wefel, Proc. 16th Int. Conf. Cosmic Rays (Kyoto) 1, 436 (1979).

66) Guzik, T. G., Ap. J. 244, 695 (1981).

67) Dwyer, R., Ap. J. 224, 691 (1978).

68) Dwyer, R. and P. Meyer, Proc. 18th Int. Conf. Cosmic Rays (Kyoto) 12, 97 (1979).

68) Fisher, A. J., F. A. Hagen, R. C. Maehl, J. F. Ormes and J. F. Arens, Ap. J. 205, 938 (1976).

70) Maehl, R. C., A. J. Fisher, F. A. Hagen and J. F. Ormes, Ap. J. (Letters) 202, L119 (1975).

71) Ferrando, P., J. J. Engelmann, P. Goret, L. Koch-Miramond, N. Petrou, A. Soutoul, N. Y. Herrstrom, B. Byrnak, N. Lund, B. Peters, I. L. Rasmussen, M. Rotenberg and N. J. Westergaard, Astron. Astrophys. 193, 69 (1988)

72) In order to plot the results for $\mathrm{Mg}$ and $\mathrm{Si}$ from reference 71 (which measured mean masses) the observed overabundance was assumed to be equally distributed between both neutron-rich isotopes.

73) Freier, P. S., J. S. Young and C. J. Waddington, Ap. J. 240, L53 (1980).

74) Young, J. S., P. S. Freier, C. J. Waddington, N. R. Brewster and R. K. Fickle, Ap. J. 240, 1014 (1981).

75) Webber, W. R., Proc. 17th Int. Conf. Cosmic Rays (Paris) 2, 80 (1981).

76) Webber, W. R., private communication (1988).

77) The effect of cross section uncertainties on the GCRS abundances was stimated by assuming a $25 \%$ uncertainty in the correction for secondary contributions.

78) Mewaldt, R. A., Rev. Geophys. and Sp. Phys. 21, 295 (1983).

79) Mewaldt, R. A., 18th Tezas Symp. on Relativiatic Astrophyoics (World Scientific, Singapore), ed. M. Ulmer, 573 (1987).

80) Simpson, J. A., Ann. Rev. Nuel. Part. Sci. 33, 323 (1983).

81) Meyer, J. P., Proc. 19th Int. Cosmic Ray Conf. (La Jolla) 9, 141 (1985).

82) Cameron, A. G. W., Essays in Nuclear Astrophysics, Proceedings of the Fowler Conf., edited by C. A. Barnes, D. D. Clayton and D. N. Schramm, Cambridge University Press, 23 (1982).

83) Wefel, J. P., private communication (1988).

84) Woosley, S. E., Astrophys. Sp. Science 39, 103 (1976).

85) Hainebach, K. L., E. B. Norman and D. N. Schramm, Ap. J. 203, 245 (1976).

86) Mewaldt, R. A., Proc. 17th Internat. Coomic Ray Conf. (Paris) 13, 49 (1981).

87) Goret, P., J. J. Engelmann, L. Koch-Miramond, J. P. Meyer, N. Lund, I. L. Rasmussen and C. Perron, Proc. 17th Internat Cosmic Ray Conf. (Paris) 9, 122 (1981).

88) Webber, W. R., Proc. 18th Internat. Cosmic Ray Conf. (Bangalore) 9, 151 (1983). 
89)Meyer, J. P., in Origin and Distribution of the Elements, ed. Mathews, G. J. (World Scientific, Singapore), 310 (1988).

90) Preszler, A. M., J. C. Kish, J. A. Lezniak, G. Simpson and W. R. Webber, Proc. 14th Int. Conf. Cosmic Rays (Munich) 12, 4096 (1975).

91) Wiedenbeck, M. E., D. E. Greiner, F. S. Bieser, H. J. Crawford, H. H. Heckman and P. J. Lindstrom, Proc. 16th Internat. Cosmic Ray Conf. (Kyoto) 1, 412 (1979).

92) Meyer, J.-P., Les elements et leurs isotopes dans $l$ univers, 22nd Liege Astrophysical Sy mposium, (University of Liege Press), 153, (1979).

93) Letaw, J. R., Ap. J. (Letters) 317, L69 (1987).

94) Guzik, T. G., J. P. Wefel and J. J. Beatty, Proc. 20th Int. Cosmic Ray Conf. (Moscow) 9, 226 (1987).

95) Woosley, S. E., private communication (1987).

96) Cesarsky, C. J., Proc. 20th Int. Cosmic Ray Conf. (Moscow) 8, 87 (1987).

97)Krombel, K. E. and M. E. Wiedenbeck, Ap. J., 328, 940 (1988).

98) Webber, W. R. and M. Gupta, Proc. 20th Int. Cosmic Ray Conf. (Moscow) 2, 129 (1987) and preprint submitted to Ap. J. (1988).

99) Grevesse, N. and E. Anders, Symposium on Cosmic Abundances of Matter (Minneapolis) AIP Conf. Proc. (1988).

100) Brenneman, H. H. and E. C. Stone, Ap. J. (Letters) 299, L57 (1985).

101) York, D. G., Ap. J. 264, 172 (1983).

102) Cummings, A. C. and E. C. Stone, Proc. 20th Int. Cosmic Ray Conf. (Moscow) 3, 413 (1987).

103) Woosley, S. E. and T.A. Weaver, Ap. J., 243, 561 (1981).

104) Casse, M. and Paul, J. A., Ap. J. 258, 860 (1982).

105) Meyer, J. P., Proc. 17th Int. Cosmic Ray Conf. (Paris) 2, 265 (1981).

106) Maeder, A., Astr. Ap. 120, 113 (1983), and 120, 130 (1983).

107)Blake, J. B. and D. S. P. Dearborn, $A d v$. Sp. Res. 4, No2-3, 89 (1984).

108) Prantzos, N., M. Arnould, J. P. Arcoragi, and Casse, M., Proc. 19th Int. Cosmic Ray Conf. (La Jolla) 3, 167 (1885).

109) Casse, M. in Composition and Origin of Cosmic Raya, ed. Shapiro, M. M. (Dordrecht: Reidel), 193 (1983).

110)Arnett, W. D., Ap. J. 168, 153 (1971).

111) Prantzos, N., C. Doom, M. Arnould and C. de Loore Ap. J. 304, 695 (1986).

112) Olive, K. A. and D. N. Schramm, Ap. J. 257, 276 (1982).

113) Audouze, J., in Proc. Nineteenth Rencontre de Moriand Astrophysics Meeting, "High Energy Astrophysies", J. Tran Thanh Van, ed., (Kim Hup Lee, Singapore), 325 (1984).

114) Garcia-Munoz, M., G. M. Mason and J. A. Simpson, Proc. 14th Int. Cosmic Ray Conf. (Munich) 1, 319 (1975).

115) Silberberg, R., M. M. Shapiro and C. H. Tsao, Proc. 14th Int. Cosmic Ray Conf. (Munich) 2, 451 (1975).

116) Audouze, J., in LAU Symposium 105, Observational Tests of Stellar Evolution Theory, eds. A Maeder and A. Renzini (Dordrecht: Reidel), 541 (1983).

117) Tosi, M., Ap. J. 254, 699 (1982).

118) Following reference 16 we would expect atmospheric corrections to subtract $\sim 0.04 \pm 0.02$ from the balloon ${ }^{2} \mathrm{H} /{ }^{4} \mathrm{He}$ measurements at $\sim 100 \mathrm{MeV} /$ nucleon in Figure 1.

119) Soutoul, A., M. Casse and E. Juliusson, Ap. J. 219, 753 (1978).

120) Koch-Miramond, L., Proc. 17th Int. Cosmic Ray Conf. (Paris) 12, 21 (1981).

121) Webber, W. R., J. C. Kish and D. A. Schrier, Proc. 20th Int. Cosmic Ray Conf. (Moscow) 2, 125 (1987). 
122)Blandford, R. D. and J. P. Ostriker Ap. J. 237, 793 (1980).

123) Axford, W. I., Proc. 17th Int. Cosmic Ray Conf. (Paris) 12, 155 (1981).

124) Wiedenbeck, M. E., private communication (1984).

125)Guzik, T. G., J. P. Wefel, H. J. Crawford, D. E. Greiner, and P. J. Lindstrom, Proc. 19th Intermat. Cosmic Ray Conf. (La Jolla) 2, 80 (1985).

126) Simon, M., W. Heinrich and K. D. Mathis, Ap. J. 300, 32 (1986).

127)Wandel, A., D. Eichler, J. R. Letaw, R. Silberberg and Tsao, C. H., Ap. J. 316, 676 (1987).

128)Ferrando, Ph. and Soutoul, A., Proc. 20th Int. Cosmic Ray Conf. (Moscow) 2, 231 (1987).

129) Gloeckler, G., Rev. Geophys. Sp. Phys. 17, 569 (1979).

130) Webber, W. R., Symposium on Cosmic Abundances of Matter (Minneapolis), AIP Conf. Proc. (1988).

131) Arnould, M., Adv. Space Res. 4, No. 2-3, 45,(1984).

132)Fisk, L. A., B. Kozlovsky and R. Ramaty, Ap. J. (Lettero) 190, L35 (1974).

133)Pesses, M. E., J. R. Jokipii and D. Eichler, Ap. J. (Letters) 246, L85 (1981).

134)Mewaldt, R. A., J. D. Spalding and E. C. Stone, Ap. J. 288, 450 (1984).

135) Mewaldt, R. A., E. C. Stone and R. E. Vogt, Ap. J., 206, 616 (1976).

136) Webber, W. R., and S. M. Yushak, Proc. 16th Internat. Cosmic Ray Conf. (Kyoto) 1, 383 (1979).

137) Smith, B. and F. B. McDonald, Proc. 17th Int. Conf. Cosmic Rays (Paris) 9, 138 (1981).

138) The Particle Astrophysics Magnet Facility ASTROMAG, eds. J. F. Ormes, M. H. Israel, M. E. Wiedenbeck, and R. A. Mewaldt, (1986).

139) Ormes, J. F., M. H. Israel, R. A. Mewaldt and M. E. Wiedenbeck, Proc. 20th Int. Cosmic Ray Conf. (Moscow) 2, 378 (1987).

140)Mewaldt, R. A., and J. F. Ormes, submitted to the Venice Conference on Physics and Astrophysics in the Space Station Era (1987). 